\title{
Evidence of host adaptation in Lawsonia intracellularis infections
}

\author{
Fabio A Vannucci ${ }^{1 *}$, Nicola Pusterla², Samantha M Mapes ${ }^{2}$ and Connie Gebhart ${ }^{1}$
}

\begin{abstract}
Background: Lawsonia intracellularis is the causative agent of proliferative enteropathy, an endemic disease in pigs and an emerging concern in horses. Enterocyte hyperplasia is a common lesion in every case but there are differences regarding clinical and pathological presentations among affected species. We hypothesize that host susceptibility to $L$. intracellularis infection depends on the species of origin of the bacterial isolate. The objective of this study was to evaluate the susceptibilities of pigs and horses to $L$. intracellularis infection using either a porcine or an equine isolate.
\end{abstract}

Materials and methods: Twelve foals and eighteen pigs were equally divided into three groups and infected with either a porcine or an equine isolate $\left(10^{9} \mathrm{~L}\right.$. Intracellularis/challenged animal), and a saline solution (negative control group). The animals were monitored regarding clinical signs, average of daily weight gain, fecal shedding of the bacteria by PCR and humoral serological response.

Results: Foals infected with the equine isolate developed moderate to severe clinical signs and maintained a lower average of weight gain compared to control foals. Fecal quantitative PCR in equine isolate-infected foals revealed higher amounts of bacterial DNA associated with longer duration of shedding compared with porcine isolateinfected foals. All four foals infected with the equine isolate demonstrated higher IgG titers in the serum compared with porcine isolate-infected foals. In the pig trial, diarrhea and seroconversion were only observed in animals infected with the porcine isolate. Pathological changes typical of proliferative enteropathy were observed in the necropsied foal infected with equine isolate and in the two necropsied pigs infected with the porcine isolate.

Conclusions: Evident clinical signs, longer periods of bacterial shedding and stronger serologic immune responses were observed in animals infected with species-specific isolates. These results show that host susceptibility is driven by the origin of the isolated L. intracellularis strain.

\section{Introduction}

Lawsonia intracellularis is an obligate intracellular bacterium and the etiologic agent of proliferative enteropathy (PE), an intestinal hyperplasic disease characterized by thickening of the mucosa of the intestine due to enterocyte proliferation [1]. The disease has been reported in a variety of animal species, including nonhuman primates, wild mammals and ratite birds [2,3]. Since the 1990s, it has been endemic in pigs and one of the most economically important diseases in the swine industry [4]. In the last decade, the disease also has been frequently reported in weanling foals worldwide, and now

\footnotetext{
* Correspondence: vannu008@umn.edu

${ }^{1}$ Department of Veterinary and Biomedical Sciences, College of Veterinary Medicine, University of Minnesota, St. Paul, MN 55108, USA

Full list of author information is available at the end of the article
}

is described as an emerging disease in the horse population [5-8].

Although hyperplasic lesions are present in every case of PE, there are some differences regarding clinical and pathological presentations among affected species. In pigs, there are two major clinical forms: a sporadic, acute, haemorrhagic diarrhea and a chronic, mild diarrhea [1]. A haemorrhagic form has also been reported in macaques but not in any other susceptible species [9]. Infected horses develop acute but non-haemorrhagic diarrhea. Furthermore, hypoproteinemia is an important clinical sign of PE in horses but it has not been reported in pigs. These observations demonstrate important hostspecific characteristics of this infection.

Isolation and cultivation of L. intracellularis has only been achieved by using dividing cells in culture under

\section{Biomed Central}


strict microaerophilic conditions. These fastidious properties restrict opportunities to study the dynamics of inter-species transmission, potential reservoirs for the bacterium and host susceptibilities to different bacterial isolates. The disease has been experimentally reproduced in hamsters, pigs and horses using species-specific isolates or intestinal homogenate derived from infected animals [10-12]. Results from cross-species experimental infections in hamsters and mice models using intestinal homogenates or porcine $L$. intracellularis isolates have consistently reproduced subclinical disease and mild lesions in infected animals [13-15]. Therefore, the bacterium seems to adapt and persist differently depending on the species origin of the isolate. The susceptibility of pigs to equine isolates or vice-versa has not been reported and may provide relevant information about host adaptation or specificity of $L$. intracellularis infections.

We hypothesize that host adaptation to L. intracellularis infection is capable of driving the susceptibilities of pigs and horses depending on the species origin of the isolate. The objective of this study was to evaluate the susceptibilities of horses and pigs to L. intracellularis infection using porcine and equine isolates. The present study reports clinical signs, longer periods of fecal shedding of bacteria and stronger serologic immune responses in pigs and foals infected with their speciesspecific isolates.

\section{Materials and methods}

\section{Challenge isolates and preparation}

The present study used a porcine (PHE/MN1-00) and an equine (E40504) L. intracellularis strain isolated from a gilt and a foal, respectively, both affected with the acute form of PE. The pathogenicity of each of these isolates was previously established in a porcine and an equine experimental model $[11,12]$. Both strains were isolated and grown in murine fibroblast-like McCoy cells (ATCC CRL 1696), as described elsewhere [16,17]. Briefly, one-day-old McCoy cells growing in $\mathrm{T}_{175}$ cell culture flasks containing Dulbecco's Modified Eagles Medium with $1 \%$ L-glutamine, $0.5 \%$ amphotericin B and $7 \%$ fetal bovine serum (FBS) were infected with $2 \mathrm{~mL}$ of L. intracellularis (with approximately $10^{6}$ organisms). Infected flasks were placed in an anaerobic jar, from which the atmospheric air was evacuated by a vacuum pump to $500 \mathrm{mmHg}$ and replaced with hydrogen gas. The infected flasks were then placed in a Tri-gas incubator with $83.2 \%$ nitrogen gas, $8.8 \%$ carbon dioxide and $8 \%$ oxygen gas and incubated with a temperature of $37^{\circ} \mathrm{C}$ for seven days [18]. After a total of ten serial cell passages in vitro, the bacteria were pelleted, suspended in sucrose-potassium glutamate $(\mathrm{pH} 7.0 ; 0.218 \mathrm{M}$ sucrose, $0.0038 \mathrm{M} \mathrm{KH}_{2} \mathrm{PO}_{4}, 0.0072 \mathrm{M} \mathrm{K}_{2} \mathrm{HPO}_{4}$ and $0.0049 \mathrm{M}$ potassium glutamate) solution with 10\% FBS and stored at $-80^{\circ} \mathrm{C}$ until the day of infection [16]. The inocula for both horse and pig experiments were identically prepared at the College of Veterinary Medicine of the University of Minnesota, using the same protocols for isolation and cultivation of $L$. intracellularis. For the horse trial, porcine and equine isolates were separately preserved in dry ice and shipped to the University of California-Davis Center for Equine Health. For both equine and porcine isolates the molecular identities were determined by multi-locus variable number tandem repeat (VNTR) analysis [19].

The number of $L$. intracellularis organisms was assessed by direct counting after immunoperoxidase staining of serial 10 -fold dilutions prepared in sterile phosphate-buffered saline (PBS) using polyclonal $L$. intracellularis-specific antibody [16]. Additionally, quantitative PCR (qPCR) was performed using an aliquot from each inoculum in order to validate the direct counting and standardize the challenge doses used in the foal and pig experimental infection models [20].

\section{Animals}

Twelve Quarter Horse foals between 4 and 5 months of age were randomly divided into three groups: Horse/ Porcine isolate $(n=4)$; Horse/Equine isolate $(n=4)$ and Horse/Negative control $(n=4)$. Similarly, 18 DurocLandrace cross pigs at 3-weeks-of-age were allocated into three groups: Pig/Porcine isolate $(n=6) ; \mathrm{Pig} /$ Equine isolate $(n=6)$ and Pig/Negative control $(n=6)$. The animals were obtained from a herd with no history of $\mathrm{PE}$ and each treatment group was housed in a different pen. Prior to study commencement, all animals were evaluated for any signs of illness by a full physical examination. In addition, blood and fecal samples from all animals were collected and tested for L. intracellularisspecific antibodies by immunoperoxidase monolayer assay (IPMA) and qPCR (described in section "Quantitative PCR and serological IgG response") in order to document the negative status for each animal. Throughout the study, the foals were housed at the University of California-Davis Center for Equine Health and fed with free choice of grass and alfalfa hay and water and were supplemented daily with a commercial foal supplement. The pigs were housed in the isolation barns at the College of Veterinary Medicine of the University of Minnesota and fed with non-medicated nursery feed and water ad libitum. Horse and pig pens were cleaned once daily. All procedures were approved by the Institutional Animal Care and Use Committees of the University of California (horse study) and University of Minnesota (pig study). The horse and pig studies were conducted in the summer of 2010 and 2011, respectively. 


\section{Experimental infection}

On the challenge day (day 0), the frozen inoculum was thawed at $37^{\circ} \mathrm{C}$ and administered within $1 \mathrm{~h}$ of thawing. The foals were sedated with detomidine hydrochloride ${ }^{a}$ $(0.01 \mathrm{mg} / \mathrm{kg} \mathrm{BWT})$ and inoculated with $50 \mathrm{~mL}$ of the inoculum via nasogastric intubation [12]. The pigs were restrained and inoculated with $30 \mathrm{~mL}$ of the inoculum using a stomach tube [16]. Control foals and pigs received sucrose-potassium-glutamate solution by the same route of inoculation.

\section{Monitoring and sample collection}

Monitoring and sample collection were conducted similarly in the horse and pig studies. All animals were observed daily for general attitude and appetite for 56 days post-inoculation (pi). Once weekly, the weight of each animal was recorded in order to determine average daily weight gains throughout the study period. Feces were collected directly from the rectum every other day and submitted for qPCR [20]. Blood samples were collected on days $0,7,14,21,28,42$ and 56 pi to determine serum concentration of total solids and for serologic analysis by IPMA [21].

Due to the non-terminal design of the horse study, foals developing either moderate to severe acute (fever, depression, anorexia, colic, diarrhea) or chronic (weight loss, peripheral edema) signs and/or hypoproteinemia $(<5.0 \mathrm{~g} / \mathrm{dL})$ were treated with doxycycline hyclate ${ }^{\mathrm{b}}$ $(10 \mathrm{mg} / \mathrm{kg}, \mathrm{PO}, \mathrm{q} 12 \mathrm{~h}$ ) for 10 days. Additional supportive treatment including intravenous flunixine meglumi$\mathrm{ne}^{\mathrm{c}}(1.1 \mathrm{mg} / \mathrm{kg}, \mathrm{IV}, \mathrm{q} 12 \mathrm{~h})$ and replacement fluids ${ }^{\mathrm{d}}$ (4$6 \mathrm{~mL}, \mathrm{IV}$, q $1 \mathrm{~h}$ ) were given based on the patient's need.

\section{Quantitative PCR and serological lgG response}

All fecal samples were analyzed by qPCR for L. intracellularis DNA at the School of Veterinary Medicine of the University of California-Davis, as described elsewhere [20]. Briefly, DNA purification was performed using an automated nucleic acid extraction system ${ }^{\mathrm{e}}$, according to the manufacturer's recommendations. Absolute quantification was calculated using a standard curve for L. intracellularis and expressed as copy numbers of the aspA gene of $L$. intracellularis per gram of feces. The standard curve was determined by using 10-fold dilutions of $L$. intracellularis derived from cell culture in McCoy cells added to $L$. intracellularis-free equine feces. Furthermore, a qPCR assay targeting a universal sequence of the bacterial 16S rRNA gene was used as quality control (i.e. efficiency of DNA purification and amplification) and as an indicator of fecal inhibition [22].

Blood samples for the collection of serum were drawn from all animals in order to determine the concentration of total solids using a refractometer and to measure anti- $L$. intracellularis specific IgG by IPMA at the
College of Veterinary Medicine of the University of Minnesota, as previously reported [21]. Positive serum samples (titer $\geq 60$ ) were tested to endpoint dilution and titers were reported as the reciprocal of the dilution.

\section{Post-mortem examination, histology and immunohistochemistry}

Two pigs from each group were euthanized on day $21 \mathrm{pi}$ and evaluated for typical PE lesions. Intestinal samples from jejunum, ileum, cecum and colon were collected, fixed in $10 \%$ buffered formalin, processed routinely for histology, embedded in paraffin, and sectioned $5 \mu \mathrm{m}$ thick. Two sections were prepared: one section was stained by haematoxylin and eosin [23] and the other by immunohistochemistry (IHC) using the streptavidin method with polyclonal antibodies to $L$. intracellularis [24]. The level of infection was assessed by IHC based on the amount of positive labeled antigen present in the intestinal sections: Grade $0=$ no positive antigen labeled; Grade $1=$ one isolated focal area of antigen labeled; Grade 2=multi-focal areas of antigen labeled; Grade $3=$ majority of the mucosa has positive antigen labeled; and Grade $4=$ all of the mucosa has positive antigen labeled [11]. Histology and IHC procedures were conducted in the Veterinary Diagnostic Laboratory of the University of Minnesota.

\section{Statistical analysis}

Descriptive analyses were used to describe clinical findings among the different groups due to the limited number of animals. Statistical analysis was performed by use of Wilcoxon-Mann-Whitney tests to assess differences in daily weight gain among the different groups. The area under curve (AUC) for the amount and duration of fecal shedding, as well as for the magnitude and duration of measureable IgG titers against $L$. intracellularis, were calculated using trapezoid rule in the SAS software (version 9.2). The Wilcoxon-Mann-Whitney test was used to compare the experimental groups based on AUC. Statistical significance was defined at values of $p<0.05$.

\section{Results}

\section{Experimental infection and pathological findings}

The challenge doses were standardized for the horse and pig trials in order to avoid any influence of dose effect on the clinical, pathological or immune response. Based on the quantification of $L$. intracellularis organisms (described in section "Challenge isolates and preparation"), which was confirmed by qPCR, each animal received $10^{9}$ bacteria intragastrically. In addition, the molecular identities of the porcine and equine isolates were confirmed after the experimental infection by VNTR typing using PCR positive samples. 
Diarrhea and significant lower daily weight gain ( $p$ $<0.05)$ were observed in pigs infected with the porcine isolate and in foals infected with the equine isolate, as shown in Table 1. Three equine isolate-infected foals developed moderate to severe clinical signs typical of equine PE, including depression, anorexia, colic and peripheral edema. Hypoproteinemia $(<5.0 \mathrm{~g} / \mathrm{dL})$ was also observed in these foals (ranging from 4.1 to $4.7 \mathrm{~g} / \mathrm{dL}$ ) between 21 and 28 days pi. Hypoproteinemia was not observed in any infected pigs. Because of the severe clinical signs, one equine isolate-infected foal was treated according to the protocol previously described, but it did not respond to antimicrobial and supportive treatment and it was then humanely euthanized 24 days pi. A full necropsy revealed severe and diffuse (from duodenum to cecum) thickening of the intestinal mucosa associated with the presence of large numbers of intracellular Lawsonia-specific antigen identified by IHC (Figure 1a and b). Based on the typical clinical signs of PE, the peak of the experimental infection occurred between the third and fourth week pi in both pigs and foals. Foals infected with the porcine isolate, pigs infected with the equine isolate and negative control groups failed to show clinical signs, hypoproteinemia (foals), lower average of weight gain or pathological changes.

The experimental infection in pigs revealed macroscopic and histologic lesions typical of porcine PE in the two animals infected with porcine isolate and euthanized 21 days pi. These lesions were associated with the presence of Lawsonia-specific antigen in the intestinal epithelium (Figure 1c and d). Neither the two pigs infected with the equine isolate nor the two negative controls which were euthanized 21 days pi showed any clinical or pathological changes (Figure 1e and f).

\section{Quantification of fecal $L$. intracellularis DNA}

Results of fecal shedding of $L$. intracellularis throughout the study period are summarized in Figure 2. Positive PCR signals for the universal bacterial 16S rRNA gene were detected in all fecal samples demonstrating the efficiency of the DNA extraction protocol. L. intracellularis DNA was observed in the feces of foals infected with the equine isolate from 12 to 38 days pi. Three foals infected with the porcine isolate shed bacteria. However, $L$.

Table 1 Average of daily weight gain (mean \pm standard error) throughout the entire study period

\begin{tabular}{llll}
\hline $\begin{array}{lll}\text { Infected } \\
\text { species }\end{array}$ & Control & \multicolumn{2}{l}{ L. intracellularis isolate } \\
\cline { 3 - 4 } & Porcine & Equine \\
\hline Pig $(n=6)$ & $0.59 \pm 0.09^{a}$ & $0.32 \pm 0.07^{b}$ & $0.54 \pm 0.11^{a}$ \\
\hline Horse $(n=4)$ & $0.90 \pm 0.04^{a}$ & $0.75 \pm 0.04^{a}$ & $-0.07 \pm 0.28^{b}$ \\
\hline
\end{tabular}

Pairwise comparisons are within columns: means that do not share letters are significantly different $(p<0.05)$. intracellularis DNA was detected in these animals at only four time points and no more than $10^{4}$ bacterial organisms per gram of feces was detected (Figure 2a). The mean areas under the "time $-L$. intracellularis organisms/g" curve (Figure 2a) were significantly different $(p<0.05)$ between foals infected with equine and porcine isolates. As a result, equine isolate-infected foals revealed higher amounts of bacterial DNA in the feces associated with longer duration of shedding compared with porcine isolate-infected foals.

In the pig trial, animals infected with the porcine isolate showed higher and longer shedding of bacteria in the feces throughout the study period $(p<0.05)$ (Figure $2 \mathrm{~b}$ ). In these animals, PCR detection of L. intracellularis lasted from day 2 to 38 pi. Two pigs infected with the equine isolate shed the bacteria at low levels $\left(10^{2}\right.$ bacteria/g of feces) on day 2 pi. All foals and pigs in the negative control group remained negative for the entire study period.

\section{Serological lgG response}

All four equine isolate-infected foals demonstrated higher IgG titers ( $\geq 3840)$ against L. intracellularis in the serum compared with the porcine isolate-infected foals $(\leq 1920)$ (Figure 3a). One foal infected with the porcine isolate showed no measurable serologic response during the entire study period. The mean areas under the time-titer curve of the IPMA titers were not significantly different $(p<0.05)$ between foals infected with equine and porcine isolates. However, because there was no serum from day 28 pi in the foal euthanized on day 21 , the area under the curve for this animal was underestimated in the analysis. Simulating IgG titers of at least 960 on days 28 and 42 for this foal, significant difference $(p<0.03)$ would be observed in the time-titer curve of the IPMA between foals infected with porcine and equine isolates.

The serologic responses in the pig experiment are summarized in Figure 3. There was no detectable serologic response in pigs infected with the equine isolate at any time point. On the other hand, the majority of porcine isolate-infected pigs demonstrated IgG titers (> 120) against $L$. intracellularis from day 21 pi. The serological responses were persistent throughout the study in foals and pigs infected with species-specific isolates. However, foals infected with the equine isolate had much stronger immune responses $(p=0.057)$ than those pigs infected with the porcine isolate.

\section{Discussion}

Marked clinical signs and pathological changes typical of PE associated with longer periods of bacterial shedding and stronger serologic immune responses were observed in pigs and foals infected with species-specific isolates. 


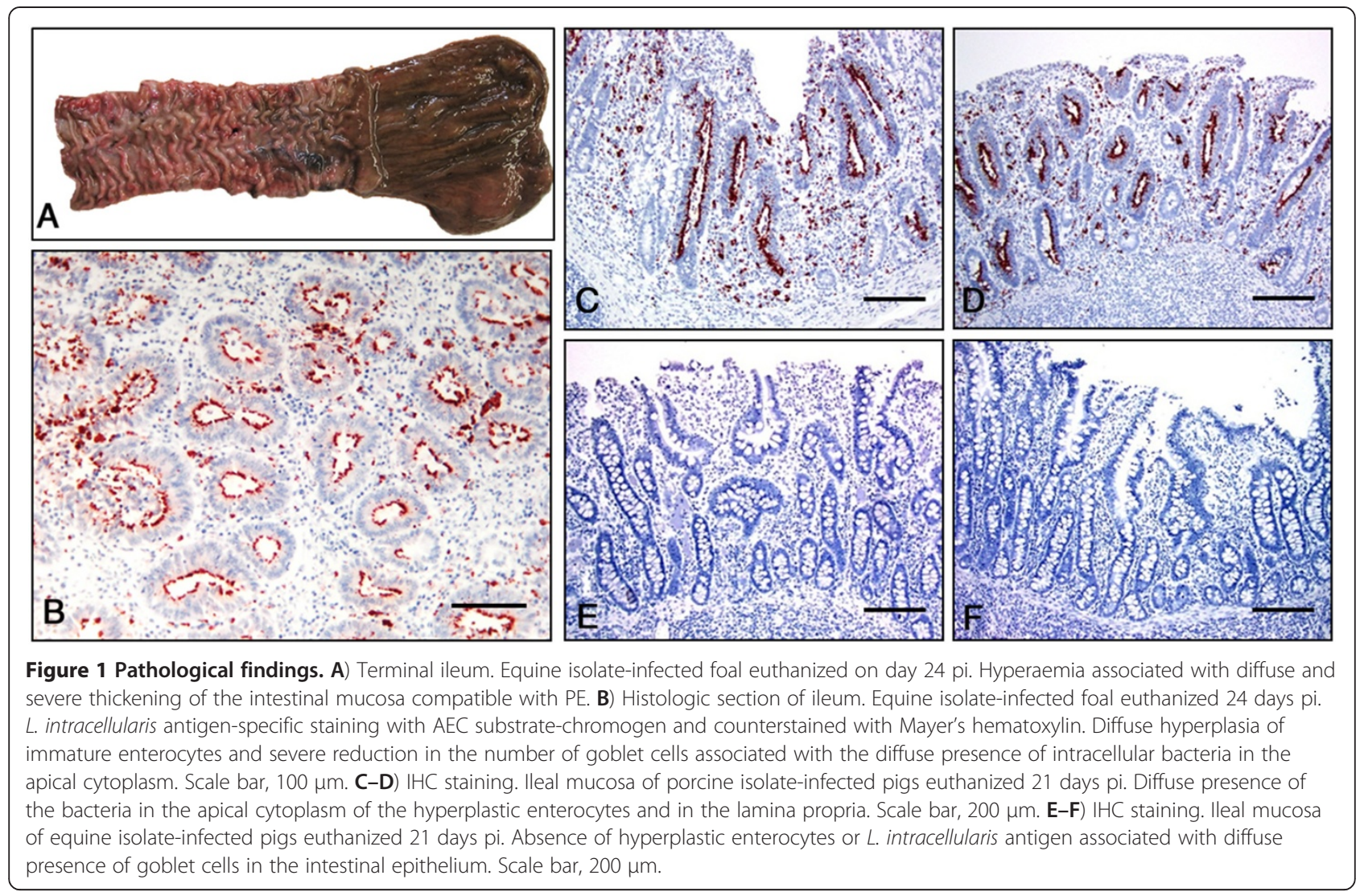

These observations were made despite the identical doses of L. intracellularis used in the horse and pig experimental infections and support the hypothesis that host susceptibilities are driven by the origin of the isolated strain. Pigs and foals demonstrated a similar pattern of fecal shedding, which lasted until 39 days pi, but the bacterial DNA was identified earlier in pigs (day 2 pi) compared to foals (day 8 pi). In those animals infected with species-specific isolates, foals exhibited a more robust serological IgG response compared with pigs which may reflect a characteristic related to the host response, regardless of the L. intracellularis isolate. Additionally, hypoproteinemia was an important sign of $\mathrm{PE}$ in the affected horses. Despite the phenotypic differences regarding the susceptibilities of these two species, gross and histological lesions characterized by thickening of intestinal mucosa due to proliferation of enterocytes were identical in those animals infected with speciesspecific isolates.

PE has been previously reproduced using pure cultures or intestinal mucosa homogenates derived from infected animals $[11,12,25]$. Since $L$. intracellularis is a fastidious organism and extremely difficult to isolate and propagate, mucosal homogenate challenge models have been reported in pigs using intestinal mucosa from PEaffected pigs [26-28]. Hamsters and mice infected with intestinal homogenates from PE-affected pigs have consistently developed subclinical PE associated with mild lesions, mainly found in the cecum and colon [15,29]. However, dehydration and profuse diarrhea were observed in hamsters inoculated with intestinal homogenate or filtrate derived from PE-affected hamsters $[10,25]$. Studies using mice experimentally infected with pure cultures of porcine L. intracellularis isolates have revealed inconsistent results. Smith et al. [14] described pathological changes in wild-type $129-\mathrm{Sv}-\mathrm{Ev}$ and IFN- $\gamma$ receptor knockout (IFN- $\gamma \mathrm{R}^{-}$) mice infected with a porcine isolate. Go et al. [30] reported gross and histological lesions in IFN- $\gamma \mathrm{R}^{-}$mice but not in three wild-type mice strains (ICR, BALB/c and C57BL/6). A more recent study showed mild histological lesions in four mice strains, including $\mathrm{BALB} / \mathrm{c}$ and $\mathrm{C} 57 \mathrm{BL} / 6$ [31]. As described above, the lack of availability of $L$. intracellularis isolates from different host species has limited the experimental infection studies to the use of porcine isolates. Recently, our research group isolated a new equine strain and its virulence was confirmed in a foal experimental model [12]. This allowed us to perform the present study which is the first cross-species experimental model using pure cultures of L. intracellularis and to evaluate different host susceptibilities and adaptation to species-specific bacterial isolates. 

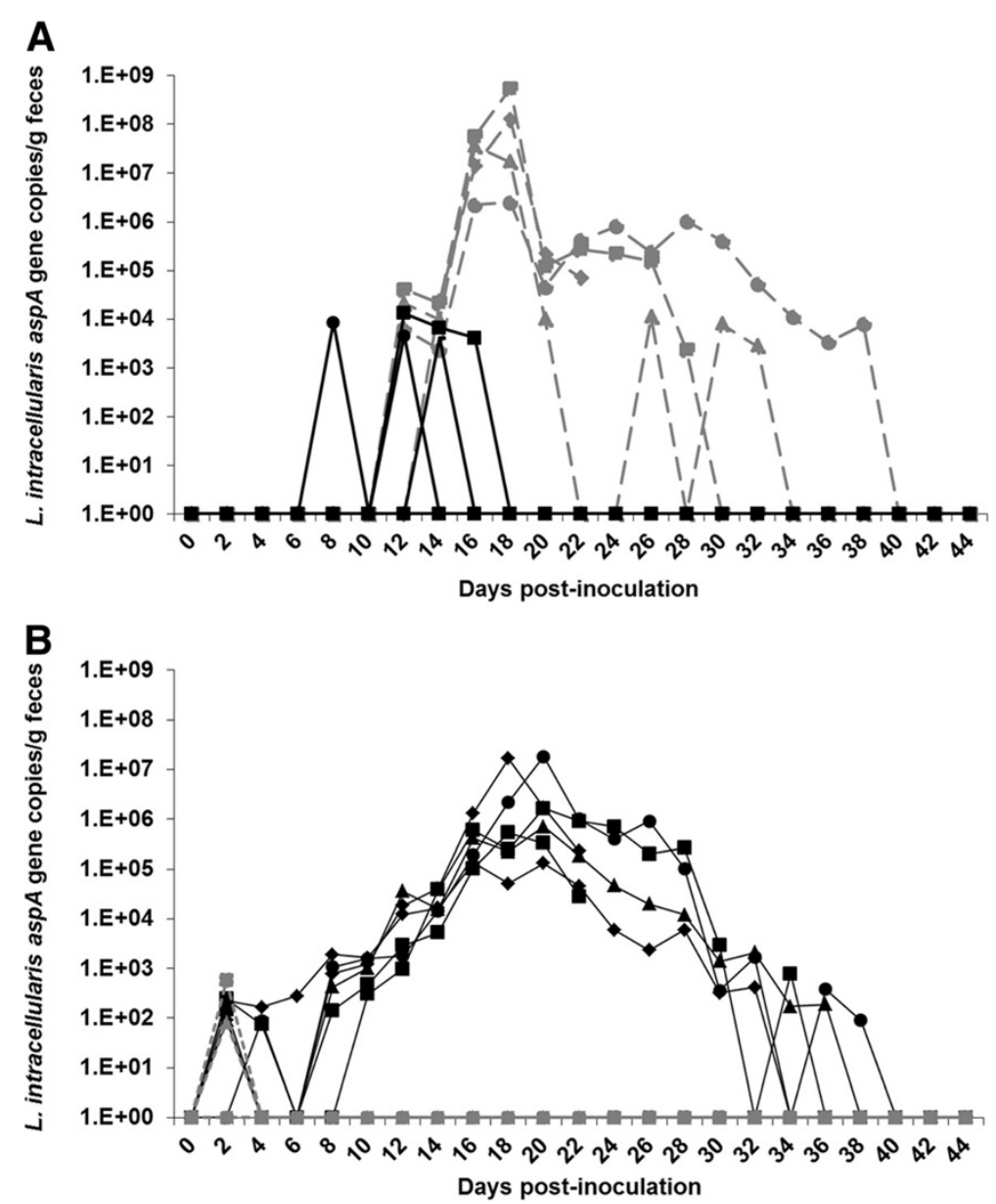

Figure 2 Quantification of the $L$. intracellularis DNA in the feces performed by qPCR. The results are expressed as copy numbers of aspA gene copies of $L$. intracellularis per gram of feces throughout the study period. A) Foal experiment. B) Pig experiment. Black-solid lines represent animals infected with the porcine isolate and grey-dashed lines represent animals infected with the equine isolate.

The challenge models previously described have contributed to advances in the prevention and control of PE by determining the efficacy of different antimicrobial drugs and vaccination protocols $[16,27,32]$. Nevertheless, little is known about the epidemiology and ecology of $L$. intracellularis infection, especially regarding interspecies transmissions, source or reservoir hosts of $L$. intracellularis and potential biological or mechanical vectors. Based on the pattern of bacterial shedding (Figure 2), foals infected with the porcine isolate and pigs infected with the equine isolate showed low levels of $L$. intracellularis DNA in the feces during a short period of time at the earlier stages of the experimental infection. Based on these findings and the uncommon opportunity of pigs and foals to share the same environment in the modern pig production system or horse breeding farms, any direct cross-species transmission in the field between these two species is unlikely.

Free-living animals have been speculated to be a reservoir of PE by allowing the maintenance of $L$. intracellularis in the wild population with successive introductions into domestic pig or horse farms. Pusterla et al. [20] detected bacterial DNA by fecal PCR in a variety of domestic and wild species, including dogs, cats, mice, rabbits, opossums, skunks and coyote, on horse breeding farms with documented occurrence of EPE. The involvement of wild animals as biological vectors for $L$. intracellularis in the pig production system has also been investigated. Using quantitative PCR, Collins et al. [33] recently identified rats trapped in endemic pig farms shedding less than $10^{5} \mathrm{~L}$. intracellularis/g of feces. However, these authors also reported that a small proportion of rats shed more than $10^{8}$ bacteria per gram of feces. Associating these observations with the results from our study leads us to speculate on the presence of different sources or reservoir species for $L$. intracellularis infections in horses and pigs. In addition, the lack of evidence supporting inter-species transmission between horses and pigs helps confirm the host adaptation to speciesspecific isolates demonstrated in the present study. 

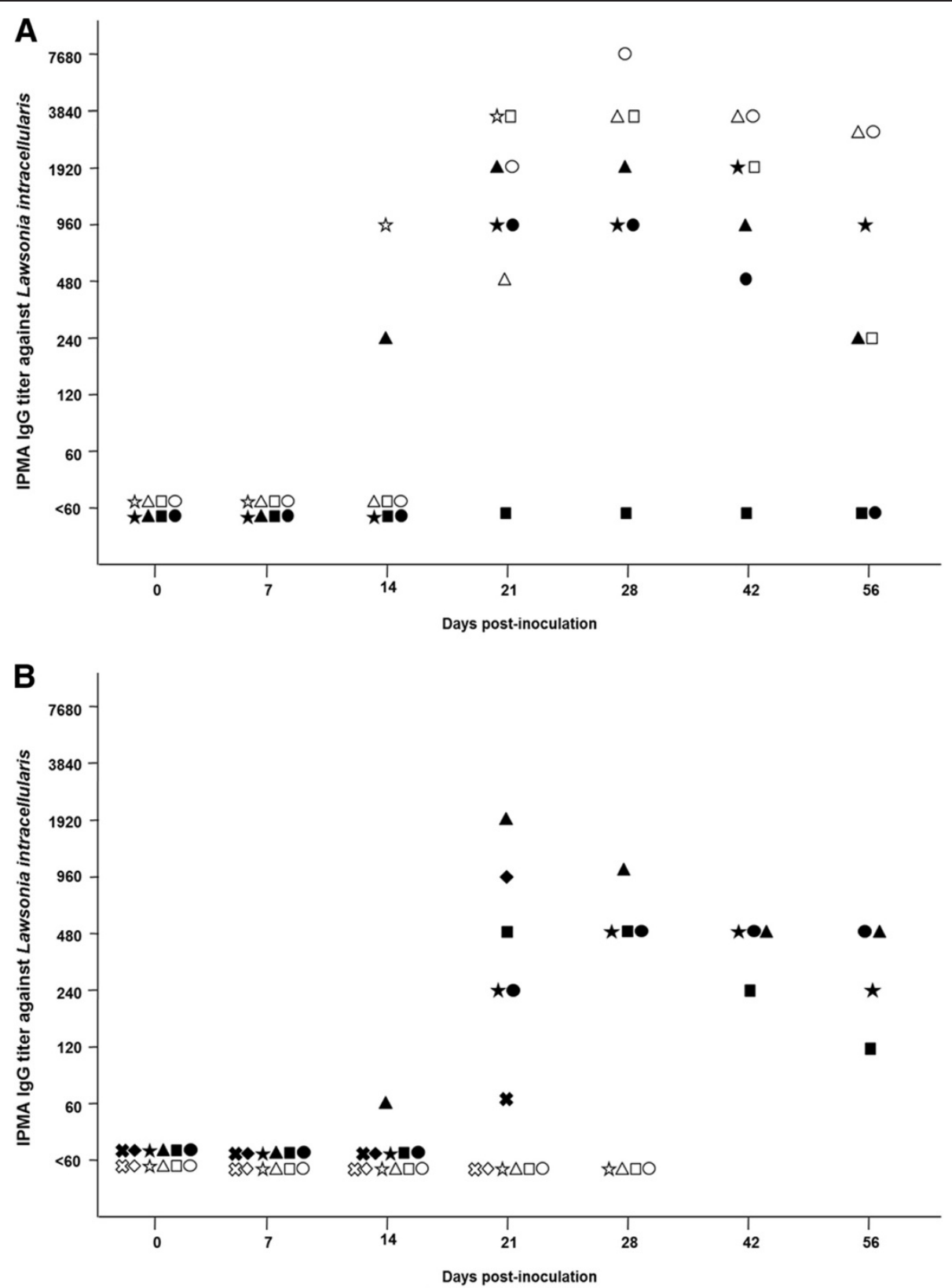

Figure 3 Serologic response against $\boldsymbol{L}$. intracellularis throughout the study period. A) Foal experiment. B) Pig experiment. White points represent animals infected with the equine isolate and black points represent animals infected with the porcine isolate.

In order to support these hypotheses, the phenotypic observations described in the present study may be linked with genotypic differences between $L$. intracellularis isolates. Comparison of the $16 \mathrm{~S}$ ribosomal DNA sequences showed a high degree of similarity among $L$. intracellularis from pigs, hamsters, deer and ostriches [34]. Four sets of primers targeting hypervariable regions of the L. intracellularis genome were used in the development of the VNTR technique [19]. This technique has shown unique and distinct VNTR profiles from epidemiologically unrelated outbreaks in pigs and horses and it was performed to confirm the molecular identity of the porcine and equine isolates used in the present study. A comprehensive analysis involving wholegenome sequencing of equine, as well as porcine, $L$. intracellularis isolates is crucial to identify any genomic variations associated with different host adaptations and susceptibilities.

In conclusion, the present study demonstrated marked clinical signs, longer periods of bacterial shedding and stronger serologic immune responses in foals and pigs experimentally infected with their species-specific 
isolates. This evidence of host adaptation in these species suggests that host susceptibilities to PE are driven by the origin of the L. intracellularis isolate. Comparative genomic analysis is a promising route to associate phenotypic characteristics with potential genomic variations between porcine and equine isolates and may help to characterize species-specific $L$. intracellularis strains and potentially novel bacterial subspecies or genotypes.

\section{Endnotes}

${ }^{a}$ Dormosedan, Pfizer Animal Health, Exton, Pa.

${ }^{\mathrm{b}}$ Doxycycline, Reva Pharmaceuticals, Sellersville, Pa.

${ }^{\mathrm{c}}$ Banamine, Schering-Plough Animal Health, Union, $\mathrm{Nj}$.

d Plasma-Lyte A, Baxter Healthcare Corporation, Deerfield, Il.

e CAS-1820 X-tractor Gene, Corbett Life Science, Sydney, Australia.

\section{Abbreviations}

PE: Proliferative enteropathy; FBS: Fetal bovine serum; VNTR: Variable number tandem repeat; PBS: Phosphate-buffered saline; IPMA: Immunoperoxidase monolayer assay; pi: Post-inoculation; QPCR: Quantitative PCR

IHC: Immunohistochemistry; AUC: Area under curve; IFN- $\gamma R^{-}$: IFN- $\gamma$ receptor knockout.

\section{Competing interests}

The authors declare they have no competing interests.

\section{Authors' contributions}

Conceived and designed the experiments: FAV, NP, CG. Performed the experiments and analyzed the data: FAV, NP, SMM, CG. Wrote the paper: FAV, NP. Coordinated and helped to draft the manuscript: CG. All authors read and approved the final manuscript.

\section{Acknowledgements}

F. A. Vannucci was supported by the Brazilian government sponsoring agency "Conselho Nacional de Desenvolvimento Científico e Tecnológico" (CNPq). This project was supported by the Center for Equine Health with funds provided by the State of California pari-mutuel fund and contributions by private donors.

\section{Author details}

'Department of Veterinary and Biomedical Sciences, College of Veterinary Medicine, University of Minnesota, St. Paul, MN 55108, USA. ${ }^{2}$ Department of Veterinary Medicine and Epidemiology, School of Veterinary Medicine, University of California, Davis, CA 95616, USA.

Received: 4 April 2012 Accepted: 25 May 2012

Published: 20 June 2012

\section{References}

1. Gebhart CJ, Guedes RMC: Lawsonia intracellularis. In Pathogenesis of Bacterial Infections in Animals. 4th edition. Edited by Gyles CL, Prescott JF, Songer JG, Thoen CO. Ames, lowa, USA: Blackwell Publishing; 2010:503-509.

2. Cooper DM, Swanson DL, Gebhart CJ: Diagnosis of proliferative enteritis in frozen and formalin-fixed, paraffin-embedded tissues from a hamster, horse, deer and ostrich using a Lawsonia intracellularis-specific multiplex PCR assay. Vet Microbiol 1997, 54:47-62.

3. Lafortune M, Wellehan JF, Jacobson ER, Troutman JM, Gebhart CJ, Thompson MS: Proliferative enteritis associated with Lawsonia intracellularis in a Japanese macaque (Macaca fuscata). J Zoo Wildl Med 2004, 35:549-552.

4. Lawson GH, Gebhart CJ: Proliferative enteropathy. J Comp Pathol 2000, 122:77-100

5. Stämpfli $\mathrm{H}$, Oliver OE: Chronic diarrhea and weight loss in three horses. Vet Clin N Am Equine Pract 2006, 22:e27-e35.
6. Frazer ML: Lawsonia intracellularis infection in horses: 2005-2007. J Vet Intern Med 2008, 22:1243-1248.

7. Guimarães-Ladeira CV, Palhares MS, Oliveira JS, Ramirez MA, Guedes RM: Faecal shedding and serological cross-sectional study of Lawsonia intracellularis in horses in the state of Minas Gerais, Brazil. Equine Vet J 2009, 41:593-596.

8. Lavoie JP, Drolet R: Equine proliferative enteropathy: an emerging disease of foals. Equine Vet Educ 2009, 21:183-185.

9. Klein EC, Gebhart CJ, Duhamel GE: Fatal outbreaks of proliferative enteritis caused by Lawsonia intracellularis in young colony-raised rhesus macaques. J Med Primatol 1999, 28:11-18.

10. Jacoby RO: Transmissible ileal hyperplasia of hamsters. I. Histogenesis and immunocytochemistry. Am J Pathol 1978, 91:433-450.

11. Guedes RM, Gebhart CJ: Comparison of intestinal mucosa homogenate and pure culture of the homologous Lawsonia intracellularis isolate in reproducing proliferative enteropathy in swine. Vet Microbiol 2003, 93:159-166.

12. Pusterla N, Wattanaphansak S, Mapes S, Collier J, Hill J, Difrancesco M, Gebhart C: Oral infection of weanling foals with an equine isolate of Lawsonia intracellularis, agent of equine proliferative enteropathy. $J$ Vet Intern Med 2010, 24:622-627.

13. Jasni S, McOrist S, Lawson GH: Reproduction of proliferative enteritis in hamsters with a pure culture of porcine ileal symbiont intracellularis. Vet Microbiol 1994, 41:1-9.

14. Smith DG, Mitchell SC, Nash T, Rhind S: Gamma interferon influences intestinal epithelial hyperplasia caused by Lawsonia intracellularis infection in mice. Infect Immun 2000, 68:6737-6743.

15. Vannucci FA, Borges EL, de Oliveira JS, Guedes RM: Intestinal absorption and histomorphometry of Syrian hamsters (Mesocricetus auratus) experimentally infected with Lawsonia intracellularis. Vet Microbiol 2010 145:286-291.

16. Guedes RM, Gebhart CJ: Onset and duration of fecal shedding, cellmediated and humoral immune responses in pigs after challenge with a pathogenic isolate or attenuated vaccine strain of Lawsonia intracellularis. Vet Microbiol 2003, 91:135-145.

17. Wattanaphansak S, Gebhart C, Olin M, Deen J: Measurement of the viability of Lawsonia intracellularis. Can J Vet Res 2005, 69:265-271.

18. Lawson GH, McOrist S, Jasni S, Mackie RA: Intracellular bacteria of porcine proliferative enteropathy: cultivation and maintenance in vitro. $J$ Clin Microbiol 1993, 31:1136-1142.

19. Beckler DC, Kapur V, Gebhart CJ: Molecular epidemiologic typing of Lawsonia intracellularis. In Proceedings of the Conference of Research Workers in Animal Disease. Edited by Ellis RP. Chicago, IL: Blackwell Publishing; 2004:124.

20. Pusterla N, Mapes S, Rejmanek D, Gebhart C: Detection of Lawsonia intracellularis by real-time PCR in the feces of free-living animals from equine farms with documented occurrence of equine proliferative enteropathy. J Wild Dis 2008, 44:992-998.

21. Guedes RM, Gebhart CJ, Deen J, Winkelman NL: Validation of an immunoperoxidase monolayer assay as a serologic test for porcine proliferative enteropathy. J Vet Diagn Investig 2002, 14:528-530

22. Mapes S, Rhodes DM, Wilson WD, Leutenegger CM, Pusterla N: Comparison of five real-time PCR assays for detecting virulence genes in isolates of Escherichia coli from septicaemic neonatal foals. Vet Rec 2007 , 161:716-718.

23. Luna LC: Manual of Histologic Staining. Methods of the Armed Forces Institute of Pathology. 3rd edition. New York: McGraw-Hill; 1968:258.

24. Guedes RM, Gebhart CJ: Preparation and characterization of polyclonal and monoclonal antibodies against Lawsonia intracellularis. $J$ Vet Diagn Investig 2003, 15:438-446.

25. Jacoby RO, Osbaldiston GW, Jonas AM: Experimental transmission of atypical ileal hyperplasia of hamsters. Lab Anim Sci 1975, 25:465-473.

26. Guedes RM, Winkelman NL, Gebhart CJ: Relationship between the severity of porcine proliferative enteropathy and the infectious dose of Lawsonia intracellularis. Vet Rec 2003, 153:432-433.

27. McOrist S, Smith SH, Shearn MF, Carr MM, Miller DJ: Treatment and prevention of porcine proliferative enteropathy with oral tiamulin. Vet Rec 1996, 139:615-618.

28. Guedes RM, França SA, Machado GS, Blumer MA, da Costa Cruz EC: Use of tylvalosin-medicated feed to control porcine proliferative enteropathy. Vet Rec 2009, 165:342-345. 
29. Murakata K, Sato A, Yoshiya M, Kim S, Watarai M, Omata Y, Furuoka H: Infection of different strains of mice with Lawsonia intracellularis derived from rabbit or porcine proliferative enteropathy. J Comp Pathol 2008, 139:8-15.

30. Go YY, Lee JK, Ye JY, Lee JB, Park SY, Song CS, Kim SK, Choi IS: Experimental reproduction of proliferative enteropathy and the role of IFN-gamma in protective immunity against Lawsonia intracellularis in mice. $J$ Vet $\mathrm{SCi}$ 2005, 6:357-359.

31. Viott A, Vannucci F, Oliveira J, Costa M, Gebhart C, Guedes R: Susceptibility of different mouse strains to Lawsonia intracellularis infection using intestinal mucosa homogenate and pure culture. In Proceedings of the $21^{\text {st }}$ Congress of International Pig Veterinary Society 2010. Edited by D'Allaire Sylvie, Friendship Robert. Vancouver, Canada: Pig Veterinary Society; 2010.

32. McOrist S, Morgan J, Veenhuizen MF, Lawrence K, Kroger HW: Oral administration of tylosin phosphate for treatment and prevention of proliferative enteropathy in pigs. Am J Vet Res 1997, 58:136-139.

33. Collins AM, Fell S, Pearson H, Toribio JA: Colonisation and shedding of Lawsonia intracellularis in experimentally inoculated rodents and in wild rodents on pig farms. Vet Microbiol 2011, 150:384-388.

34. Cooper DM, Swanson DL, Barns SM, Gebhart CJ: Comparison of the $16 \mathrm{~S}$ ribosomal DNA sequences from the intracellular agents of proliferative enteritis in a hamster, deer, and ostrich with the sequence of a porcine isolate of Lawsonia intracellularis. Int J Syst Bacteriol 1997, 47:635-639.

doi:10.1186/1297-9716-43-53

Cite this article as: Vannucci et al:: Evidence of host adaptation in

Lawsonia intracellularis infections. Veterinary Research 2012 43:53.

\section{Submit your next manuscript to BioMed Central and take full advantage of:}

- Convenient online submission

- Thorough peer review

- No space constraints or color figure charges

- Immediate publication on acceptance

- Inclusion in PubMed, CAS, Scopus and Google Scholar

- Research which is freely available for redistribution 